\title{
A general model of price competition
}

\author{
with soft capacity constraints*
}

\section{Marie-Laure Cabon-Dhersin† Nicolas Drouhin ${ }^{\ddagger}$}

April 8, 2019

\begin{abstract}
We propose a general model of oligopoly with firms relying on a two factor production function. In a first stage, firms choose a certain fixed factor level. In the second stage, firms compete on price, and adjust the variable factor to satisfy all the demand. When the factors are substitutable, the capacity constraint is "soft", implying a convex cost function in the second stage. We show that there exists a continuum of subgame perfect equilibria in pure strategies, whatever the returns to scale. Among them a payoff-dominant one can always be selected. The equilibrium price may increase with the number of firms.
\end{abstract}

Key words: price competition, tacit collusion, convex cost, capacity constraint, limit pricing strategy, returns to scale.

Code JEL: L13, D43

${ }^{*}$ We thank for their helpful comments and suggestions the participants of PET 2017, Paris, EARIE 2017, Maastricht, AFSE 2017, XXXIII Jornadas de Economia Industrial, Barcelona, and various invited seminars, and also Marc Deschamps, Raffaele Fiocco, Robert Gary-Bobo, Yukio Koriyama, Laurent Linnemer, Jean-Pierre Ponssard, Robert Somogyi.

${ }^{\dagger}$ Université de Rouen Normandie, CREAM, Address: 3 avenue Pasteur, 76100 Rouen, France. (marie-laure.cabon-dhersin@univ-rouen.fr)

${ }^{\ddagger}$ Corresponding author at Ecole Normale Supérieure Paris-Saclay, Ens Paris-Saclay 61 avenue du Président Wilson, 94230 Cachan, France. (nicolas.drouhin@ens-paris-saclay.fr) 


\section{Introduction.}

This article investigates price competition between a variable number of capacity constrained firms producing a homogeneous good. In our model, firms rely on a production function with two substitutable factors that are chosen sequentially. The first factor, chosen in a first stage, remains "fixed" in the second during which firms compete on price and adjust the second "variable" factor to match their demand. In this setting, the fixed factor can be seen as a capacity. This means that firms in our model are capacity constrained but that this constraint is "soft" because they can always increase production beyond their optimal capacity, albeit at an increasing marginal production cost. Our results are general: the production function can be of any form and returns to scale do not have to be constant or decreasing.

The results are as follows. There is a continuum of subgame perfect Nash Equilibria in pure strategies in which all firms operate the market at a high level of price, generalizing the seminal one stage model of Dastidar $(1995,2001)$ in a two stage setting. A payoff-dominant subgame perfect Nash Equilibrium can always be selected.

Moreover: 1) Because the short-run cost function is convex whatever the returns to scale, the existence of an equilibrium for the whole game is disentangled from the nature of the returns to scale, a result that has never been reported. 2) In the first stage, there is a threshold for the fixed factor below which competitors can adopt limit pricing strategies in the second stage, a property that will discipline the equilibrium price, especially when the number of firms is low. 3) The equilibrium price appears to increase with the number of firms, a theoretical result that has seldom been reported when information is perfect (Rosenthal, 1980; Chen and Riordan, 2008; Gabaix et al., 2016) and never been explained relying on supply-side argument. 


\section{Related literature}

This paper bridges three lines of literature, price competition with convex cost initiated by Dixon $(1987,1990)$ and fully characterized by Dastidar (1995), the BertrandEdgeworth constrained capacity approach to price competition, and the literature on capacities and limit pricing strategies.

In his seminal model of price competition, Joseph Bertrand (1883) considered interactions between two firms that have identical linear cost functions and simultaneously set their prices. According to this model, even if the number of competing firms is small, price competition leads to a perfectly competitive outcome in a market for an homogeneous good. The unique equilibrium price equals the firms' (constant and common) marginal cost and each firm's profit is equal to zero. This result is referred as the Bertrand Paradox. For a long time, following Edgeworth's 1925 initial insight, the belief was that there was a serious equilibrium existence problem (in pure strategies) when considering decreasing returns to scale and/or convex cost functions. However, Dastidar (1995) proved that a continuum of pure strategy Nash equilibria in price competition does exist when costs are strictly convex. In this model, following Chamberlin (1933), firms are assumed to match all incoming demand at the price that is announced. As noted by Yano and Komatsubara (2017), this Dastidar-Chamberlin specification is increasingly recognized as an important stream of literature. ${ }^{1}$ This assumption can be justified by the existence of hidden cost for turning part of the demand away (Dixon, 1990). ${ }^{2}$ An other justification can be that, in many regulated market, the authority obliged the firms to match all the incoming demand, with the idea that this rule will protect the consumer (Spulber, 1989). It is thus interesting to understand how those regulated market functions.

\footnotetext{
${ }^{1}$ See, among many others, Dastidar (1995, 2001, 2011b); Hoernig (2002); Chaudhuri (1996); Chowdhury (2009, 2002); Vives (1999); Chowdhury and Sengupta (2004); Novshek and Chowdhury (2003); Bagh (2010); Routledge (2010).

${ }^{2}$ In markets with "drastic" capacity constraint (see below) the choice of turning part of the consumer away is natural consequence of the choice of capacity in the first stage. In Dixon (1990) the choice of the price and the choice of the quantity that will be offered to answer part of this demand are simultaneous.
} 
With this assumption, a firm undercutting its rivals will attract all the demand but, when the cost function is convex, this move may not necessarily be profitable. At equilibrium therefore, prices may be higher than the average cost and even higher than the marginal cost. Dastidar (2001) shows that, when the costs are sufficiently convex, the collusive outcome may even be an equilibrium. On the contrary, with strictly subadditive costs and symmetric firms, it can be shown that there is no equilibrium in price competition (Dastidar, 2011b). ${ }^{3}$ The source of subadditivity can be either increasing returns to scale or the existence of fixed costs when variable unitary costs are constant or not too convex (Hoernig, 2007; Baye and Kovenock, 2008; Saporiti and Coloma, 2010). In this Bertrand-Dastidar approach to price competition it is the convexity of the cost function that resolves the Bertrand Paradox. As mentioned in the introductory paragraph, in our model, the convexity of the short-run cost function in the second stage is due to the decreasing marginal productivity of the variable factor. Our model thus shares the main properties of the Dastidarian framework, and that, whatever the returns to scale.

As pointed out by Vives (1999), following Edgeworth (1925), there is a long tradition in Industrial Organization to solve the Bertrand Paradox by considering that firms are constrained by their production capacities when matching the incoming demand. In the modern literature, this argument has been put forward by Kreps and Scheinkman (1983), among others. In a two-stage game, they obtain that quantity pre-commitment, in the first stage, and price competition, in the second, sustain the Cournot outcome provided the constrained capacities are not "too high". As shown by Davidson and Deneckere (1986), this result is sensitive to the choice of a rationing rule for the residual demand (see Vives, 1999, p.124, for details). This result is built on "drastic" capacity constraints, that is, the marginal cost of production in excess of capacity is infinite. Our approach relies on the same type of two stage game with capacity chosen in the

\footnotetext{
${ }^{3}$ Dastidar (2011a) introduces asymmetric cost functions and proves that, in this case, when the monopoly break-even prices differ, an equilibrium can be found even if costs are strictly subadditive.
} 
first. However, the softness of the capacity constraint induces a smoother cost function in the second stage. Less-rigid capacity constraints have been introduced previously in a number of studies (see Maggi, 1996; Boccard and Wauthy, 2000, 2004; Chowdhury, 2009, for example) directly in the cost function. Cabon-Dhersin and Drouhin (2014) have established a rigorous basis for such "soft" capacity constraints starting from the microeconomic production function and production factors chosen sequentially. Burguet and Sákovics (2017) follow the same approach, but with a very different model of price competition in the second stage, whereby firms can tailor their prices to each consumer.

Beyond the issue of price competition, strategic investment capacity decisions are also a very classical question in industrial organization with regard to entry deterrence (Spence, 1977; Dixit, 1980, among many others). In this kind of model, choosing an excess capacity in the first stage drives away potential competitors. In our model, all the competitors are already operating in the market. However, a firm that chooses too low a capacity in the first stage will be unable to match its competitors' prices profitably in the second. Firms must therefore choose a high enough capacity to avoid limiting their pricing strategies in the second stage.

In this context, we propose a model of price competition with "soft" capacity constraints that extends in many dimensions Cabon-Dhersin and Drouhin (2014). We adopt a very general, non parametric, production function instead of a Cobb-Douglas one. Second, we consider the possibility for the returns to scale to be decreasing or increasing instead of being only constant. Third, we consider an oligopoly instead of duopoly, allowing the effects on the market price of the number of firms to be investigated. Notably, we find that when there are few firms in the market, the equilibrium price can increase when new firms enter whatever the returns to scale. While the concept of price-increasing competition is not new to the literature (Rosenthal, 1980; Chen and Riordan, 2008; Gabaix et al., 2016), our model highlights a simple explanation for this phenomenon: cost convexity combined with endogenous capacities induces a "ca- 
pacity effect" that can offset the negative effect on prices of additional competing firms. When more firms operate in the market, the level of the fixed factor (capacity) tends to decrease and the stronger convexity of the cost function increases the equilibrium price.

The paper is organized as follows. Section 2 rigourously characterizes the notion of "soft" capacity constraints; the complete model is solved in Section 3; in Section 4 finally, a general method for numerical simulations is presented along with a "textbook example" to illustrate some interesting properties.

\section{Characterization of soft capacity constraints}

Firms produce a homogeneous good using the same technology represented by a two factor production function. The factors are chosen sequentially. We denote $z$ the level of the factor chosen in the first stage (the fixed factor) and $v$, the level of the factor (the variable factor) chosen in the second stage. We denote $y$ the level of production, and $f: \mathbb{R}_{+}^{2} \rightarrow \mathbb{R}_{+}$, giving:

$$
y=f(z, v)
$$

The only assumptions are that $z$ is an essential production factor $(f(0, v)=0$ for all $v$ ) and $f$ increases with $z$ and $v$, shows decreasing marginal factor productivity, and is quasi-concave. Thus: $f_{z}>0, f_{v}>0, f_{z z}<0, f_{v v}<0$ and $-f_{z z} f_{v}^{2}+2 f_{z v} f_{v} f_{z}-f_{v v} f_{z}^{2}>0$. It is important to emphasize that we make no general assumptions about the nature of the returns to scale or the level of substitutability between the two production factors. When $z$ is fixed, Equation (1) defines the variable factor as an implicit function of $z$ and $y, \hat{v}(y, z)$.

Lemma 1. 1) The function $\hat{v}$ is quasi-convex and fulfils:

$$
\hat{v}_{y}(y, z)=\frac{1}{f_{v}(z, v)}>0
$$




$$
\begin{gathered}
\hat{v}_{z}(y, z)=-\frac{f_{z}(z, v)}{f_{v}(z, v)}<0 \\
\hat{v}_{y y}(y, z)=-\frac{f_{v v}(z, v) \hat{v}_{y}(y, z)}{f_{v}(z, v)^{2}}>0 \\
\hat{v}_{z z}(y, z)=\frac{-f_{z z} f_{v}^{2}+2 f_{z v} f_{v} f_{z}-f_{v v} f_{z}^{2}}{f_{v}(z, v)^{3}}>0 \\
\hat{v}_{y z}(y, z)=\hat{v}_{z y}(y, z)=-\frac{f_{v z}(z, v)+\hat{v}_{z}(y, z) f_{v v}(z, v)}{f_{v}(z, v)^{2}}<0
\end{gathered}
$$

2) Moreover, if $f$ is (strictly) concave then $\hat{v}$ is (strictly) convex.

Proof: In the Appendix A.1

We can therefore define the cost as a function of $(y, z)$. With $w_{1}$, the price of factor $z$ and $w_{2}$, the price of the factor $v$, we have:

$$
C(y, z)=\underbrace{w_{1} z}_{F C(z)}+\underbrace{w_{2} \hat{v}(y, z)}_{V C(y, z)}
$$

Setting the level of the fixed factor corresponds to choosing a capacity. In this model, it is possible to match any incoming demand but at an increasing marginal cost. It is in this way that the capacity constraint is" soft". The sequential choice of production factors implies that the cost function is convex, whatever the returns to scale. Thus, when firms compete on price in the second stage, our model inherits the general properties of the Dastidarian framework.

It is noteworthy that as always, the fixed cost depends on the level of the fixed factor, but so does the variable cost. The level chosen for the capacity will have qualitative implications for the shape of the variable cost function. Models that start from an arbitrary cost function usually miss this effect.

Finally, it is important to notice that the softness of the capacity constraint comes from the substitutability of the production factor. As pointed out by Cabon-Dhersin and Drouhin (2014, p. 428) and Burguet and Sákovics (2017), if the production factors are fully complementary (Leontief technology), our approach is equivalent to the usual 
"drastic" capacity constraint.

\section{Equilibrium of the game}

Firms first set their fixed factor to a certain level then compete on price in a second stage (they can observe the level of fixed factor played by other firms in the first stage). The demand of the whole market is continuous, twice differentiable, decreasing and admits a choke-price ${ }^{4}$

$$
D: \mathbb{R}^{+} \longrightarrow \mathbb{R}^{+} \text {with } D^{\prime}(p) \leq 0, D\left(p_{\max }\right)=0, D(0)=Q_{\max } .
$$

The strategic variable for the firms in stage 2 is price. We denote $p_{i}$ the price of firm $i$ and $\vec{p}=\left(p_{1}, . ., p_{n}\right)$, the vector of prices of all the $n$ firms in the market. We denote $p_{L}=\operatorname{Min}\left\{p_{1}, . ., p_{n}\right\}$ and we define the set $M=\left\{j \in\{1, . ., n\} \mid p_{j}=p_{L}\right\}$. We denote $m=\operatorname{Card}(M)$ the number of firms quoting the lowest price. Firms have to supply all the demand they face in stage 2 at price $p_{i}$. The demand function of firm $i$ is defined as follows:

$$
D_{i}(\vec{p})= \begin{cases}0 & \text { if } p_{i}>p_{L} \\ \frac{D\left(p_{i}\right)}{m} & \text { if } p_{i}=p_{L}\end{cases}
$$

We can now express the profit $\pi_{i}$ of each firm $i$, when $m$ firms operate in the market (set the lowest price).

$$
\pi_{i}\left(\vec{p}, z_{i}\right)=p D_{i}(\vec{p})-w_{1} z_{i}-w_{2} \hat{v}\left(D_{i}(\vec{p}), z_{i}\right)
$$

\footnotetext{
${ }^{4}$ A shown by Saporiti and Coloma (2010), Table 1, pp 4-5, the assumption of a choke-price is very standard in the literature. It is especially the case in the Dastidarian tradition with convex costs in which the assumption facilitates the proof of the existence of the thresholds in price that will be crucial in the reasoning. The main drawback is that it prevents the use of the simple iso-elastic demand function. However, in the standard case of Bertrand competition with constant marginal cost, Baye and Morgan (1999), in opposition with Harrington (1989), proved that the assumption can be critical: "when the absence of choke-price gives rise to an unbounded monopoly profit", any positive (but finite) payoff vector can be achieved in a symmetric Nash equilibrium.
} 


$$
\pi_{i}\left(\vec{p}, z_{i}\right)= \begin{cases}-w_{1} z_{i} & \text { if } p_{i}>p_{L} \\ p \frac{D(p)}{m}-w_{1} z_{i}-w_{2} \hat{v}\left(\frac{D(p)}{m}, z_{i}\right) \stackrel{\text { def }}{=} \hat{\pi}\left(p, z_{i}, m\right) & \text { if } p_{i}=p_{L}=p\end{cases}
$$

The function $\hat{\pi}\left(p, z_{i}, m\right)$ represents the profit of firm $i$ when $m$ firms (including firm i) quote the lowest price, $p$. This function depends on the level of the fixed factor set in the first stage of the game. The assumptions described above for the production function are sufficient to ensure that $\hat{\pi}_{z z}<0$. However, even if the profit is necessarily concave in $y$, this does not guarantee that $\hat{\pi}$ is strictly concave in $p$. This occurs if the demand function is not too concave or too convex:

$$
-\frac{D^{\prime}(p)^{2}}{m} \frac{\hat{v}_{y y}}{\hat{v}_{y}}\left(\frac{D(p)}{m}, z\right)<D^{\prime \prime}(p)<-2 \frac{D^{\prime}(p)}{p}
$$

The left-hand side of the inequality corresponds to the sufficient condition for the short run cost to be convex in $p$ and the right-hand side is the sufficient condition for the revenue function to be concave in $p .^{5}$ Moreover, if we want this condition to hold whatever the number of firms, in particular when this number grows to infinity, the left-hand side should tend to zero and the demand function has to be convex (nonstrictly).

To solve the equilibrium of the game in stage 2 , the first step is to test whether firm $i$ can deviate profitably from an outcome in which $m$ firms (including firm $i$ ) quote the same price. The following two thresholds are defined with this purpose in mind. The first, $\bar{p}$, is the maximum price for which firms cannot increase their profits by unilaterally lowering their prices. The second, $\hat{p}$, is the minimum price for which firms cannot increase their profits by unilaterally increasing their prices. In the traditional Bertrand competition model with constant average/marginal costs, these two thresholds are equal and correspond to the unique equilibrium of the game, implying marginal cost pricing. In our more general setting with a convex short-run

\footnotetext{
${ }^{5}$ This is a standard assumption to make, even if when starting directly from a cost function, it is hidden within the general assumption that the profit function is concave in $\mathrm{p}$.
} 
cost function, as in Dastidar (1995), the two thresholds are never equal and define a price interval for each firm for which there is no profitable deviation.

Let us start by studying a firm's ability to increase its profit by undercutting its competitors. For that purpose we define, for $m \geqslant 2$, the function $\Omega(p, z, m) \stackrel{\text { def }}{=}$ $\hat{\pi}(p, z, 1)-\hat{\pi}(p, z, m) . \Omega$ can be interpreted as the incentive for a firm to lower its price when the market price is $p$. Thus when $\Omega \leq 0$, it is not profitable for the firm to lower its price while when $\Omega>0$, it is profitable for the firm to do so.

Lemma 2. For a given $z$ and $m$, there is a unique threshold $\bar{p}(z, m) \in\left(0, p_{\max }\right)$ that solves $\Omega(p, z, m)=0$

When $p \leq \bar{p}, \Omega(p, z, m) \leq 0$ and when $p>\bar{p}, \Omega(p, z, m)>0$

Proof: In the Appendix A.2

$\bar{p}(z, m)$ is the highest price with no incentive to deviate when $m$ firms operate the market. It can be shown that this price is necessarily higher than the marginal cost (it follows directly from inequality (16) in the Appendix A.2).

We can now study the possibility for a firm to increase its profit by increasing its price. This case is much simpler because in the second stage, the fixed cost, $w_{1} z$, is sunk, and the firm is only motivated to produce if the variable part of the profit is positive. If this is not the case at the current price, $p$, increasing the price will induce zero demand for the firm and thus zero production and will reduce its losses.

Lemma 3. For a given $z$ and $m \geq 1$, there is a unique $\hat{p}(z, m)$ in the interval $\left(0, p_{\max }\right)$ for which: $\hat{\pi}(\hat{p}, z, m)=-w_{1} z$ Moreover, $\hat{\pi}(\hat{p}, z, m)$ decreases with $m$ and $\bar{p}(z, m)>\hat{p}(z, m)$.

Proof: In the Appendix A.3

For a given $z, \hat{p}(z, m)$ is the minimum price that motivates production in the second stage when $m$ firms operate in the market.

The price interval $[\hat{p}(z, m), \bar{p}(z, m)]$ is crucial to solving for the equilibrium of the 
game in stage 2 . These prices will have to be compared with the purely collusive price, $p^{*}$, when $m$ firms operate in the market.

Lemma 4. For a given $z$ and $m \geq 1$, there is a unique $p^{*}(z, m)$ in the interval $\left(0, p_{\max }\right)$ for which $p^{*}(z, m) \stackrel{\text { def }}{=} \underset{p}{\arg \max }\{\hat{\pi}(p, z, m)\}$

Moreover, $p^{*}(z, m)>\hat{p}(z, m)$.

\section{Proof: In the Appendix A.4}

A simple interpretation is that this is the collusive price when all firms chose to set their fixed factor to the same level in the first stage (when $m=1$, it is the monopoly price). We will see that $p^{*}$ can fall within $[\hat{p}(z, m), \bar{p}(z, m)]$, but not necessarily.

Lemma 5. $\forall m \in[1, n], \hat{p}(z, m), \bar{p}(z, m)$ and $p^{*}(z, m)$ are strictly decreasing in $z$ and $m$ over their respective domains.

\section{Proof: In the Appendix A.5}

At this point, it is important to remark that, in the following, $z$ will be endogenous, while $m$ will remain exogenous, and that, every things else being equal, a higher number of firms will tend to diminish the demand for each firm and thus the level of fixed factor required to match it. Thus Lemma 5 describes, at the same time, the direct effect of a higher $m$ on all the three thresholds in price, $\hat{p}, \bar{p}, p *$ and an indirect effect through the endogenous diminution of $z$ that will play in the opposite direction. Moreover, it is obvious that the effect of a unitary increase in the number of firms on each firm's demand, and thus on $z$, will be much higher when this number is low, as well as the intensity of the indirect effect.

We have now gathered all the elements required to characterize the equilibrium prediction for the whole game.

Each firm $i$ chooses a pure strategy $\left(z_{i}, p_{i}\right)$ in $\mathbb{R}^{+} \times\left[0, p_{\text {max }}\right]$, played sequentially. We are searching for outcomes of the game $(\vec{z}, \vec{p})$ in $\mathbb{R}^{+n} \times\left[0, p_{\text {max }}\right]^{n}$ that are Subgame Perfect Nash Equilibrium (SPNE) in which all the $n$ firms operate in the market at the same price. For doing that we rely only on the definition of Nash equilibrium, and 
check for the non existence of profitable deviation in every subgames and for the whole game (cf. Methodological statement in the Appendix B).

Proposition 1. The three following conditions are necessary for an outcome of the game $(\vec{p}, \vec{z})$ in which all the $n$ firms operate in the market at the same price $p^{N}$ to be a SPNE:

1. Optimal level of fixed factor: All $n$ firms set their fixed factor to the same level, $z^{N}=z^{*}\left(p^{N}, n\right)$, with $z^{*}(p, n)$ being a solution of the program:

$$
\mathcal{P}_{1}(p, n)\left\{\begin{array}{l}
\max _{z} \hat{\pi}(p, z, n) \\
\text { s.t. } p \leq \bar{p}(z, n)
\end{array}\right.
$$

2. Profitability: $\hat{\pi}\left(p^{N}, z^{N}, n\right) \geq 0$

\section{Non-existence of limit pricing strategies:}

$\hat{\pi}\left(p^{N}, z^{N}, n\right) \geq \hat{\pi}\left(\hat{p}\left(z^{N}, n\right), \underset{z}{\operatorname{argmax}} \hat{\pi}\left(\hat{p}\left(z^{N}, n\right), z, 1\right), 1\right)$

\section{Proof: In the Appendix B.1}

Each condition corresponds to a type of deviation in the whole game (a deviation that can concern both $z$ and $p$ ). Among those possible deviations, condition 1 focused on unidimensional deviations in $z$, i.e. deviations that can preserve the same price as an equilibrium in the second stage. Because of the multiplicity of equilibria in the second stage, a same equilibrium price can be sustained by a continuum of $z$. Of course, among this continuum only the $z$ that maximizes the profit will be non-dominated by that kind of deviation. That is the spirit of program $\mathcal{P}_{1}(p, n)$, the constraint of this program delimiting the right bound of the continuum. It means that, when the constraint is binding, the capacity $z$ solution of program $\mathcal{P}_{1}(p, n)$ will be lower than the optimal $z$ of the unconstrained program.

Program $\mathcal{P}_{1}(p, n)$ plays a central role in all the reasoning of this article. An important property of the program is that its solution is always unique, thanks to the strict 
concavity of the profit function $\hat{\pi}$ with regard to $z$. Thus, for a given number of firms, $n$, there exists a bi-univocal decreasing relation between $z$ and $p$, i.e. $z^{*}(p, n)$. Only couple in which $p \in\left[0, p_{\max }\right]$ and $z=z^{*}(p, n)$ satisfies condition 1. and SPNE are to be searched within the reduced set of outcomes satisfying this condition for all players. The associated profit for each firm will thus be:

$$
\Pi\left(p^{N}, n\right) \stackrel{\text { def }}{=} \pi\left(p^{N}, z^{*}\left(p^{N}, n\right), n\right)
$$

Condition 2 is quite simple, a firm playing $z=0$ and $p=p_{\max }$ will always obtain zero profit. So an outcome that brings negative profit will always be eliminated by that kind of deviation when considering the whole game.

Finally condition 3 considers possible deviations, in which a firm massively invests in the fixed factor, and sets, in the second stage, a low price that will not be sustainable by rivals ${ }^{6}$. That kind of deviations will be especially profitable for outcomes characterized by a low level of fixed factor for all firms. It is particularly the case for outcomes fulfilling condition 1 , when the constraint of program $\mathcal{P}_{1}(p, n)$ is binding.

A comparison can be made with models in which firms choose simultaneously the price and the capacity as in Dixon (1990). In these models, firms choose the price and the capacity that maximizes the profit for this price without any constraint. In our model, the sequential nature of the game adds a constraint to the program that will be binding when the considered price is "high". For a given $z, p^{N}$ has to be a Nash Equilibrium in the second stage, implying that $z$ cannot be "too high". That's the spirit of Condition 1. As stated before, when the constraint is binding, the level of fixed factor is sub-optimal, high prices can only be sustained with a lower level of fixed factor. But if the level of fixed factor is too low, then limit pricing strategies become possible, excluding the possibility for this outcome to be SPNE of the game. That's the Spirit of condition 3. We will see in the calibrated textbook example of

\footnotetext{
${ }^{6}$ Cabon-Dhersin and Drouhin (2014) overlooked the possibility of limit pricing strategies in the model, and thus was unable to identify the effect.
} 
Section 4, that, due to this "capacity effect", an increase of the number of firm may change this balance. The threshold in price above which the constraint of program $\left(\mathcal{P}_{1}\right)$ is binding will increase with the number of firms, limiting the profitability of limit pricing strategies. Thus potentially higher prices may be sustained as equilibria when the number of firms increases.

It is important to notice that Proposition 1 characterises only some necessary conditions for an outcome of the game to be a SPNE.

Proposition 2. An outcome of the game that fulfils all three necessary conditions of Proposition 1 and such that:

$$
\forall p \in\left[\hat{p}\left(z^{N}, n\right), p^{N}\right), \Pi\left(p^{N}, n\right) \geq \Pi(p, n)
$$

is a SPNE of the whole game.

Proof: In the Appendix B.2.

Proposition 2 considers the remaining possible deviations in which a firm deviates by increasing its level of fixed factor slightly, but sufficiently to exclude the initial level of price $p^{N}$ from the range of Nash equilibria in the second stage. But to associate a profit to that kind of deviation, firms should be able to predict what equilibrium will be selected in the second stage. For allowing that, it will be convenient to complete Subgame-perfection by some mechanism of equilibrium selection, or assumptions on firms beliefs or expectations to predict what will happen in the second stage.

However, when the condition of Proposition 2 is fulfilled, whatever the possible Nash selected in the second stage when considering that kind of unilateral deviation, profit is lower. And, if firms do not coordinate, profit is also lower for the deviating firm. We can thus conclude that in this special case, that corresponds to prices that are associated with the increasing part of the function $\Pi$ we do not nead supplementary assumption to obtain a SPNE. When the condition is not fulfilled, corresponding to price associated with the strictly decreasing part of the function $\Pi$. Subgame perfection 
is not powerful enough to conclude.

Proposition 3. The unique outcome in which all $n$ firms choose the same fixed factor level, $z^{C}$, in the first stage and quote the same price, $p^{C}$, in the second, with $p^{C}$ being a solution of the program,

$$
\left(\mathcal{P}_{2}\right) \begin{cases} & \max _{p} \hat{\pi}(p, z, n) \\ \text { s.t. } \quad & z^{C} \stackrel{\text { def }}{=} z=\operatorname{argmax}\left\{\mathcal{P}_{1}(p, n)\right\} \\ & \hat{\pi}(p, z, n) \geq 0 \\ & \hat{\pi}(p, z, n) \geq \hat{\pi}(\hat{p}(z, n), \underset{z}{\operatorname{argmax}} \hat{\pi}(\hat{p}(z, n), \tilde{z}, 1), 1)\end{cases}
$$

is a SPNE of the game.

Moreover, $\hat{\pi}\left(z^{C}, p^{C}\right)$ is the Payoff Dominant SPNE of the game. More generally, it dominates all the outcomes that satisfy the necessary conditions of Proposition 1.

Proof: Because all firms have the same technology, it is obvious that any $(p, z)$ that fulfil the constraints of Program $\mathcal{P}_{2}$, also fulfils the three necessary constraints of Proposition 1. Moreover, thanks to the envelop theorem, the solution of program $\mathcal{P}_{2}$, also fulfils the supplementary condition of Proposition 2. Because, of the strict concavity of the profit function $\hat{\pi}$ with regards to $p$, stemming from Equation (8), this solution is unique. It follows that, by construction, $\left(p^{C}, z^{C}\right)$ is the Payoff Dominant SPNE.

In the remainder of the article, we will consider $\left(p^{C}, z^{C}\right)$ to be the unique and symmetric predictable outcome of the price competition game with soft capacity constraints. As pointed out by Cabon-Dhersin and Drouhin (2014), the solution of program $\left(\mathcal{P}_{2}\right)$ is collusive by nature (i.e. it corresponds to a joint profit maximisation program). When neither of the constraints on programs $\left(\mathcal{P}_{1}\right)$ and $\left(\mathcal{P}_{2}\right)$ are binding, the predictable outcome will verify $p^{C}=p^{*}\left(z^{C}, n\right)$, with $p^{*}$ defined as in Lemma 4 . In this case the predictable outcome is fully collusive. When the constraint on Program $\left(\mathcal{P}_{1}\right)$ is binding (i.e. no profitable deviation in stage $2, p \leq \bar{p}(z, n)$ ), then $p^{C}=\bar{p}\left(z^{C}, n\right)$, a price that is 
necessarily higher than marginal cost, as noted in the comment of Lemma 2. For this reason we will name this case "weakly collusive", because this situation is characterized by a mark-up that is strictly positive but lower than in the "fully collusive case". However, as proved in Proposition 3, this "collusive" solution is a Subgame Perfect Nash equilibrium, a result that is very unusual in a non-repeated game.

It should be emphasized however, that, beside the more general setting of the problem, the strategy for proving the result in the present paper is also completely different. Cabon-Dhersin and Drouhin (2014) solve the continuum of Nash equilibria in the second stage, then apply a first time payoff dominance, then move backward to the resolution of the first stage, and apply for a second time payoff dominance. In this paper we solve directly the whole game for the $S P N E$ and then apply payoff dominance once. The method is not only more parsimonious, but it allows to understand that the collusive nature of the predictable outcome is not a simple tautology resulting from the use of payoff dominance, but is a natural extension of program $\left(\mathcal{P}_{1}\right)$, a necessary condition for having a SPNE.

\section{A textbook example}

The model in this article is built on very general assumptions: two substitutable factors chosen sequentially, a quasi-concave production function, decreasing marginal factor productivity. Propositions (1) and (3) show that the equilibrium prediction for the whole game can be seen as a solution to a maximisation program subject to three different inequality constraints. We can exploit this unusual and remarkable property to compute equilibrium predictions of parametrical versions of price competition models with soft capacity constraints. 


\subsection{General procedure and parametrization}

In this section, we provide a general procedure to compute equilibria and apply it to solve a simple parametrical example numerically as a "textbook case", assuming a Cobb-Douglas production function and linear demand.

The production function is:

$$
f(z, v)=A\left(z^{1-\alpha} v^{\alpha}\right)^{\rho}
$$

with $\rho>0$, the scale elasticity of production, and, $\rho \alpha<1$ and $\rho(1-\alpha)<1$ because of the decreasing marginal factor productivity. In general of course, the Cobb-Douglas production function is quasi-concave. It will be concave when $\rho=1$ (constant returns to scale) and strictly concave when $\rho<1$ (decreasing returns to scale). Taking $y=f(z, v)$, the function $\hat{v}$ is easily obtained by direct calculation:

$$
\hat{v}(y, z)=\frac{y^{\frac{1}{\alpha \rho}}}{A^{\frac{1}{\alpha \rho}} z^{\frac{1-\alpha}{\alpha}}}
$$

Thus, when $n$ firms operate in the market, the function $\hat{\pi}$ can be written:

$$
\hat{\pi}(p, z, n)=p \frac{D(p)}{n}-w_{1} z-w_{2} \frac{y^{\frac{1}{\alpha \rho}}}{A^{\frac{1}{\alpha \rho}} z^{\frac{1-\alpha}{\alpha}}}
$$

The demand function is assumed to be linear:

$$
D(p)=b\left(p_{\max }-p\right)
$$

with $b>0$. We will show that although the assumptions are simple, they are sufficient to demonstrate the full richness of our theoretical framework. We take the Payoff dominant subgame perfect Nash Equilibrium of Proposition (3) - the solution of programme $\left(\mathcal{P}_{2}\right)$ - as the predictable outcome of our general model of price competition with soft capacity constraints. But because the "non-existence of limit pricing strategies" condition can be tricky to deal with directly, we will proceed sequentially. 
Step 1. We solve program $\left(\mathcal{P}_{1}\right)$ for a given number of firms, $n$, a given price $p \in$ $\left(0, p_{\max }\right)$, and a given vector of parameters $\left(\alpha, \rho, A, b, w_{1}, w_{2}\right)$, and obtain $z^{*}(p, n)$ the level of the fixed factor that efficiently sustains price $p$. We are thus able to calculate $\Pi(p, n)=\hat{\pi}\left(p, z^{*}(p, n), n\right)$.

Step 2. For a given $n$, the process in step 1 can be repeated for any $p \in\left(0, p_{\max }\right)$. So we are able to draw $\Pi(p, n)$ as a function of $p$ point by point.

Step 3. The profitability condition $\Pi(p, n) \geq 0$ can be tested for each point $(p, \Pi(p, n))$ calculated in Step 2.

Step 4. For each point $(p, \Pi(p, n))$ calculated in Step 2, we can calculate $\hat{p}\left(z^{*}(p, n), n\right)$ and then test for the non-existence of limit pricing strategies: $\hat{\pi}\left(p, z^{*}(p, n), n\right) \geq$ $\hat{\pi}\left(\hat{p}\left(z^{*}(p, n), n\right), \underset{\tilde{z}}{\operatorname{argmax}} \hat{\pi}\left(\hat{p}\left(z^{*}(p, n), n\right), \tilde{z}, 1\right), 1\right)$.

Step 5. For each point $(p, \Pi(p, n))$ calculated in Step 2, we can check if the non profitable deviation in stage 2 condition is binding or not.

Step 6. For each point $(p, \Pi(p, n))$ calculated in Step 2, we can check if the condition of Proposition 2 is binding or not. In practice it means that all the points in the decreasing part of $\Pi(p, n)$ cannot be $S P N E$.

Step 7. Among all the $(p, \Pi(p, n))$ that satisfy the profitability test in Step 3 and the non-existence of limit pricing strategies test in Step 4, we search for the price that provides the highest profit, which is $p^{C}$, the equilibrium price solution of $\left(\mathcal{P}_{2}\right)$. If in Step 5 the non profitable deviation in stage 2 condition is binding, $p^{C}=\bar{p}\left(z^{*}\left(p^{C}, n\right), n\right)$, otherwise, $p^{C}=p^{*}\left(z^{*}\left(p^{C}, n\right), n\right)$.

For a given number of firms and all prices, the constraints of Program $\left(\mathcal{P}_{2}\right)$ can all be binding or slack. In each case, there will be a threshold price delimiting the subdomain in which each constraint is binding. We will denote: $\tilde{p}(n)=\bar{p}\left(z^{*}(p, n), n\right)$, the threshold above which profitable deviations are excluded in the second stage; $p^{0}(n)$, the threshold 
above which firms earn a positive profit; $p^{L}(n)$, the threshold above which a fixed factor can be chosen in the first stage that can make limit pricing strategies profitable in the second, and, finally, $p^{1}(n)$ the threshold above which $\Pi(p, n)$ is decreasing in $p$.

Consider the following numerical example. ${ }^{7}$ Figure 1 shows the behavior predicted for a duopoly $(n=2)$, with constant returns to scale $(\rho=1), \alpha=.7, p_{\max }=10$, and all the other parameters normalized to 1.
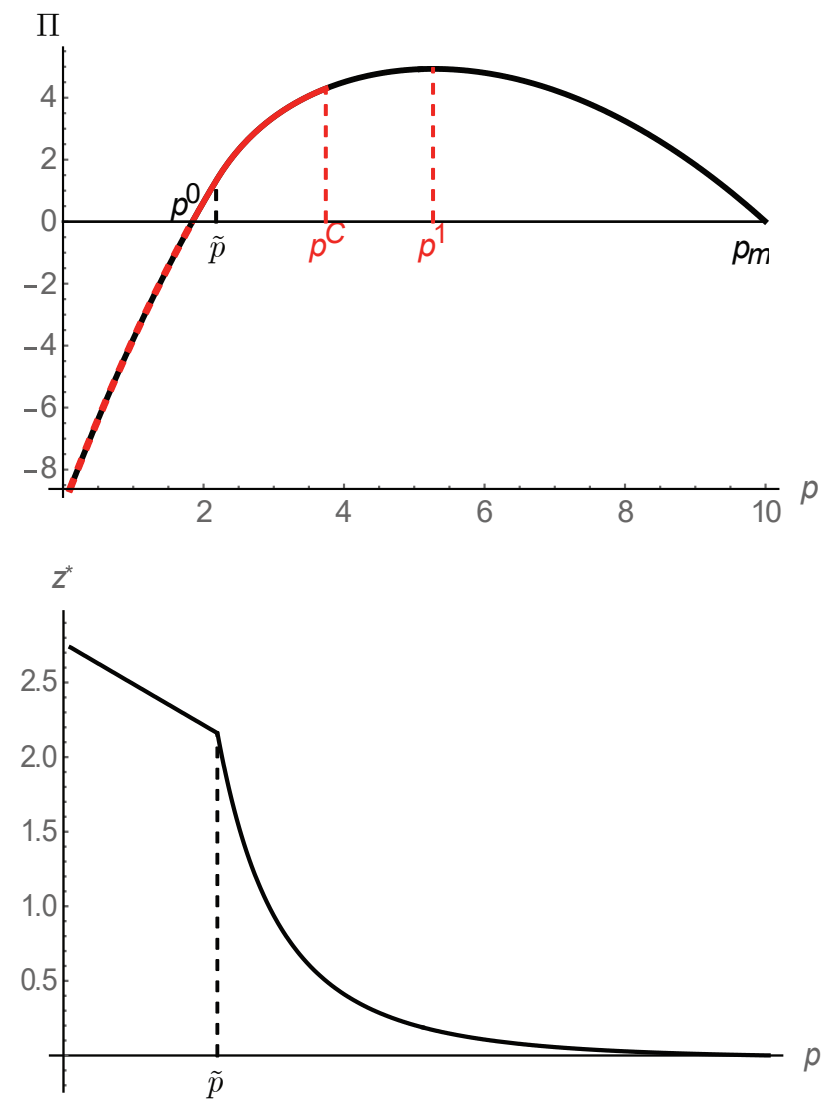

Figure 1: The SPNE interval for price (plain red) and associated fixed factor levels when $\rho=1, \alpha=.7, n=2, w_{1}=1, w_{2}=1$.

The lower graphic represents $z^{*}(p, n)$ the solution of the Program $\left(\mathcal{P}_{1}\right)$ i.e. the efficient level of the fixed factor taking into account the non profitable deviation in stage 2 constraint. For $p \leq \tilde{p}$, the constraint is slack. Of course, $z^{*}$ decreases as $p$

\footnotetext{
${ }^{7}$ Numerical simulations were performed using Wolfram Research Mathematica 11. The optimization programs were solved numerically using the NMaximize function and the value of $\hat{p}\left(z^{*}(p, n), n\right)$ was obtained using the Findroot function.
} 
increases, converging to zero as $p$ tends to $p_{\max }$. Conversely, for $p \geq \tilde{p}$, the constraint is binding $\left(p=\bar{p}\left(z^{*}, n\right)\right)$. It will become apparent that a binding constraint implies a much lower level of $z$ for a given price.

The upper graph in Figure 1 shows the whole function $\Pi(p, n)=\hat{\pi}\left(p, z^{*}(p, n), n\right)$ for $p \in\left(0, p_{\max }\right)$. The left dotted part, for $p \in\left(0, p^{0}\right)$, corresponds to negative profits. This price interval can therefore not be a SPNE of the two-stage game. The right part of the curve (in black) does not fulfil the non-existence of limit pricing strategies condition (verified in Step 4 of our procedure), and cannot correspond either to a SPNE. Consequently, the remaining (red) part of the curve corresponds to values of $p$ for which both the profitability, non-existence of limit pricing strategies and Propositon 2 conditions are fulfilled. This means that all $(p, z)$ pairs for which $p$ belongs to $\left[p^{0}, p^{C}\right]$ and $z=z^{*}(p, n)$ are SPNE of the two-stage game. It is easy to check that $p^{C}$ corresponds to the "Payoff dominant" SPNE of the whole game (the solution of program $\left(\mathcal{P}_{2}\right)$ ). With this vector of parameters, we can see that the price $p^{1}$ that maximizes $\Pi(p, n)$ does not correspond to a SPNE (note that the $\Pi$ function is defined in step 1 of our general procedure).

\section{Effect of the convexity of the variable cost}

The convexity of the cost function in the second stage is a crucial feature of our model. With a Cobb-Douglas production function, this convexity in the second stage (with $z$ fixed) is determined by the product of $\alpha$ and $\rho$. When $\alpha \rho$ tends to one, the variable cost function becomes linear. For a given level of scale elasticity, $\rho$, a lower $\alpha$ corresponds to a "more convex" production function.

Figure 2 shows the effect of different levels of convexity on the equilibrium prediction of the whole game when five firms operate in the market.

In the upper graphic, $\alpha=.45$, meaning that the variable cost function is highly convex. In this case, $\tilde{p}$ is higher. The more convex variable cost function implies that 
price deviation in the second stage is more costly (here, with five firms, the deviating firm will have to produce approximatively five times more.) Thus, $p^{C}$ and $p^{L}$ differ from $\bar{p}$, and the corresponding $z^{*}$ will be higher. This is why $p^{L}>p^{1}=p^{C}$. The maximum of $\Pi(p, n)$ corresponds to the solution of Program $(\mathcal{P} 2)$.
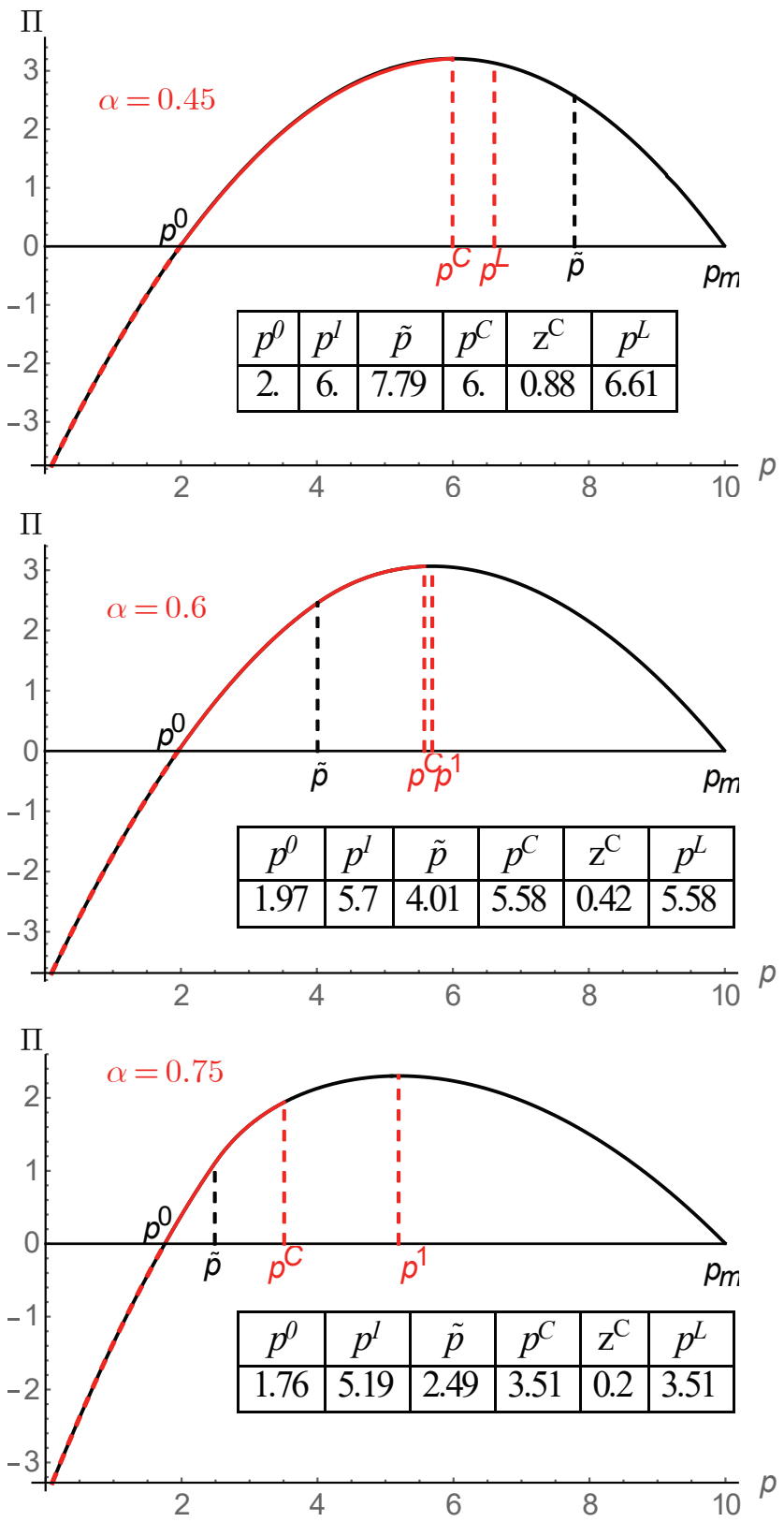

Figure 2: Effect of $\alpha$ on the $S P N E$ interval for price (plain red) ( $\rho=1, n=5, w_{1}=1$, $\left.w_{2}=1\right)$. 
In the middle graphic, with $\alpha=.6, \tilde{p}$ is now lower than $p^{1}$ and $p^{L}=p^{C} \cdot p^{1}$ and $p^{L}=p^{C}$ are thus $\bar{p}$, meaning that the non profitable deviation condition in the second stage is binding (implying that $z^{C}$ is much lower). At $p^{1}$, limit pricing strategies are profitable $\left(p^{1}>p^{L}\right) ; p^{1}$ is not a $S P N E$. This is why $p^{C}=p^{L}$ is the highest possible profit in the absence of of limit pricing strategies.

The lower graph shows that the behavior is similar with $\alpha=.75$, with $p^{C}$ being much more lower than $p^{1}$. The non-existence of limit pricing strategies constraint excludes more than half of the prices between $p^{0}$ and $p^{1}$ from being SPNE.

Finally, in this example, when $\alpha \rightarrow 0.45 \rightarrow 0.6 \rightarrow 0.75, p^{C} \rightarrow 6 \rightarrow 5.58 \rightarrow 3.51$ and $z^{C} \rightarrow 0.88 \rightarrow 0.42 \rightarrow 0.20$ : the lower the convexity is, the lower the equilibrium price is. The effect on the level of the fixed factor is more complex to analyze. A lower price implies a higher demand and thus, all things being equal, optimally requires a higher fixed factor to produce. However, a higher $\alpha$ implies that the production process uses the variable factor more intensively, and thus that $z$ is lower. Moreover, when the "non profitable deviation in the second stage" condition is binding, the optimal level of $z$ (the solution of program $\mathcal{P}_{1}$ ) is much lower (cf. Figure 1).

The same method can be used to study the effect of all parameters other than the number of firms.

\section{Effect of the number of firms and of returns to scale}

We will now study how the price varies with the number of firms. We will show that the nature of the returns to scale has a qualitative impact on this relation.

Considering constant returns to scale first, Figure 3 shows the equilibrium prediction of $p^{C}$ as a function of the number of firms when $\rho=1, \alpha=0.7, w_{1}=1$, and $w_{2}=1$. Two important thresholds are apparent. When the number of firms is low (between 2 and $n_{0}$ ), the non-existence of limit pricing strategies constraint is binding. The lower $n$ is, the more effective this constraint is, meaning that the $p^{L}(n)=p^{C}$ 
threshold increases with $n$. From $n=n_{0}$ onwards, the non-existence of limit pricing strategies constraint is no longer binding. Between $n_{0}$ and $\tilde{n}$, the non-profitable deviation constraint is binding. The corresponding prices are $\bar{p}$. The higher $n$ is, the less effective this constraint is. Prices continue to increase with $n$. Beyond $\tilde{n}$, none of the constraints are binding at equilibrium. $p^{C}$ corresponds to a purely collusive outcome. Because of the constant returns to scale, the market price $p^{C}$ is independent of the size of the firm and thus of the number of firms sharing the market.

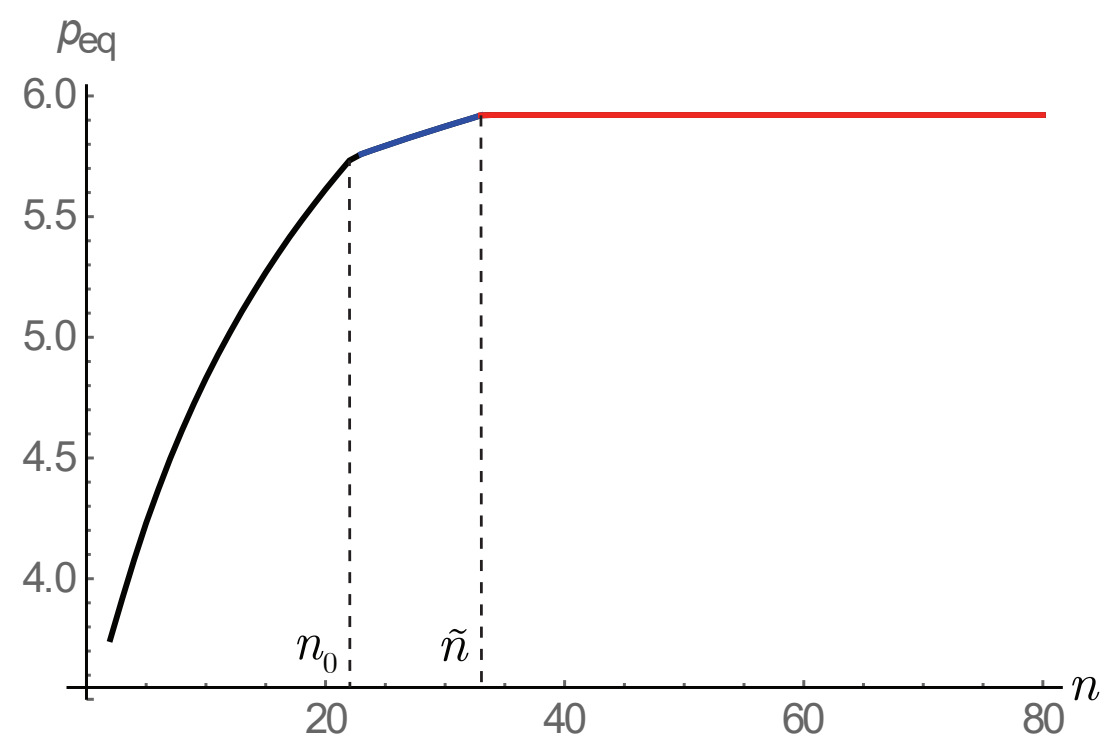

Figure 3: Effect of the number of firms on equilibrium prices $\left(\rho=1, \alpha=0.7, w_{1}=1\right.$, $\left.w_{2}=1\right)$.

This result is very unusual! When the number of firms is lower than $\tilde{n}$, the price increases with the number of firms. This is still because the cost function is convex. In our model, any firm that deviates (either in the second stage by lowering its price or in the first stage by following a limit pricing strategy in $z$ ) will capture the entire market (i.e. operate in the market alone). The increase in production is proportional to the number of firms. Because of the convexity of the variable cost, the higher the increase in production is, the lower the incentive to deviate is. However, a convex short-run cost function does not guarantee that the price increases with the number of firms. As 
shown in Lemma 5, and also by Dastidar (2001), for a given $z, \hat{p}, \bar{p}$ and $p^{*}$ decrease with $n$. It is the endogeneity of $z$, a specificity of our model, that adds a "capacity effect" that overcomes the direct effect of $n$ on $p$ for a given $z$. At equilibrium, $z$ will tend to decrease with $n$ and will, according to Lemma 5 , have an indirect positive effect on $p$. As shown in the lower part of figure 1 this effect of $z$ on $p$ dominates when the constraint $p \leq \bar{p}$ is binding.

Let us now consider the limit case in which the number of firms tends to infinity. Because of the homotheticity of the Cobb-Douglass function, the size and market share of each firm will tend to zero. However, the price will remain at the fully collusive level, significantly higher than the constant long-run average/marginal cost. The markup will remain strictly positive and, because of the constancy of the returns to scale, constant (i.e. never tending to zero). In our oligopoly model, perfect competition does not appear as an asymptotic property when the number of firms tends to infinity.

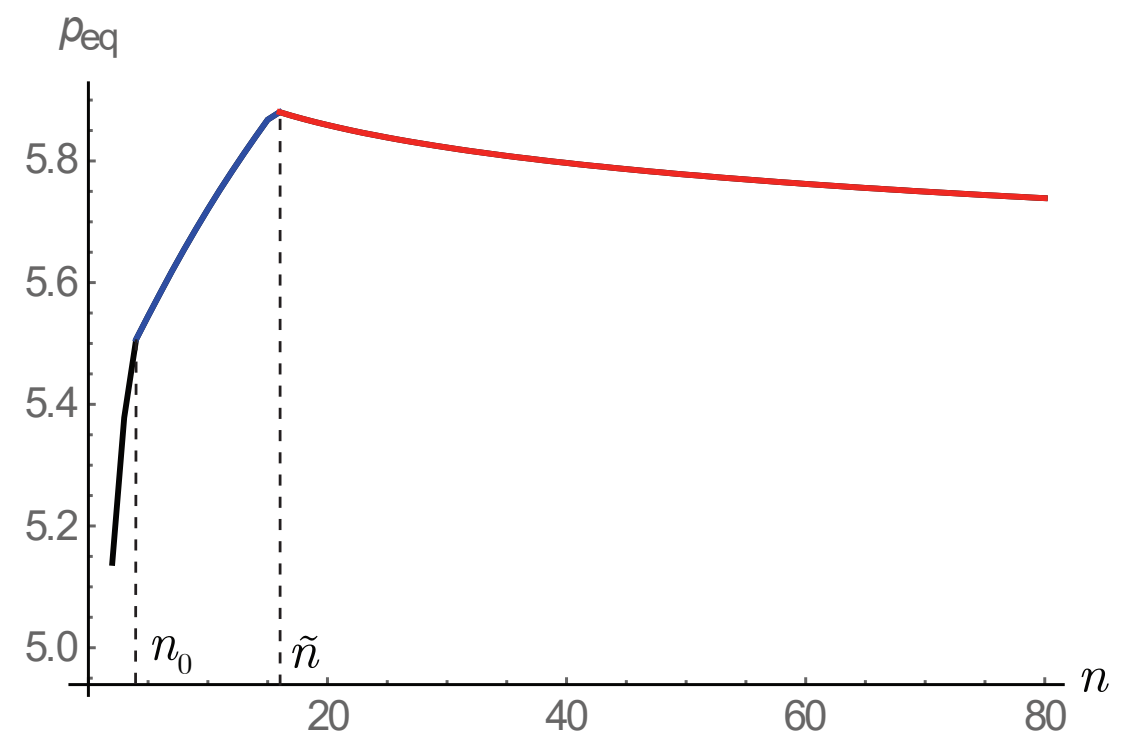

Figure 4: Effect of the number of firms on equilibrium prices $\left(\rho=0.9, \alpha=0.7, w_{1}=1\right.$, $\left.w_{2}=1\right)$.

Considering now decreasing returns to scale $(\rho=0.9)$ with all the other parameters left unchanged, the properties represented in Figure 4 are similar to those shown in 
Figure 3. The only difference is that in Figure 4 the right part of the curve (in red) decreases as the number of firms increases. This is a direct effect of the decreasing returns to scale. Smaller firms will be more efficient and will have an interest, when the outcome is purely collusive, in sustaining slightly lower prices.

Figure 5 illustrates the case of increasing returns to scale $(\rho=1.02)$. The lower envelop of the three curves on the left-hand side of the figure (up to $n_{1}$ ) follows the same trend as described above for the corresponding parts of Figures 3 and 4, with the purely collusive part (in red, between $\tilde{n}$ and $n_{1}$ ) being slightly increasing because of the increasing returns to scale (a greater number of smaller firms sharing the market is less efficient). The novelty is that beyond the $n_{1}$ threshold, the non-existence of limit pricing strategies constraint becomes binding again. Limit pricing strategies are more efficient because of the increasing returns to scale. Beyond $n_{1}$, this gain is sufficient to cancel the effect of the convexity of the variable cost function described previously.

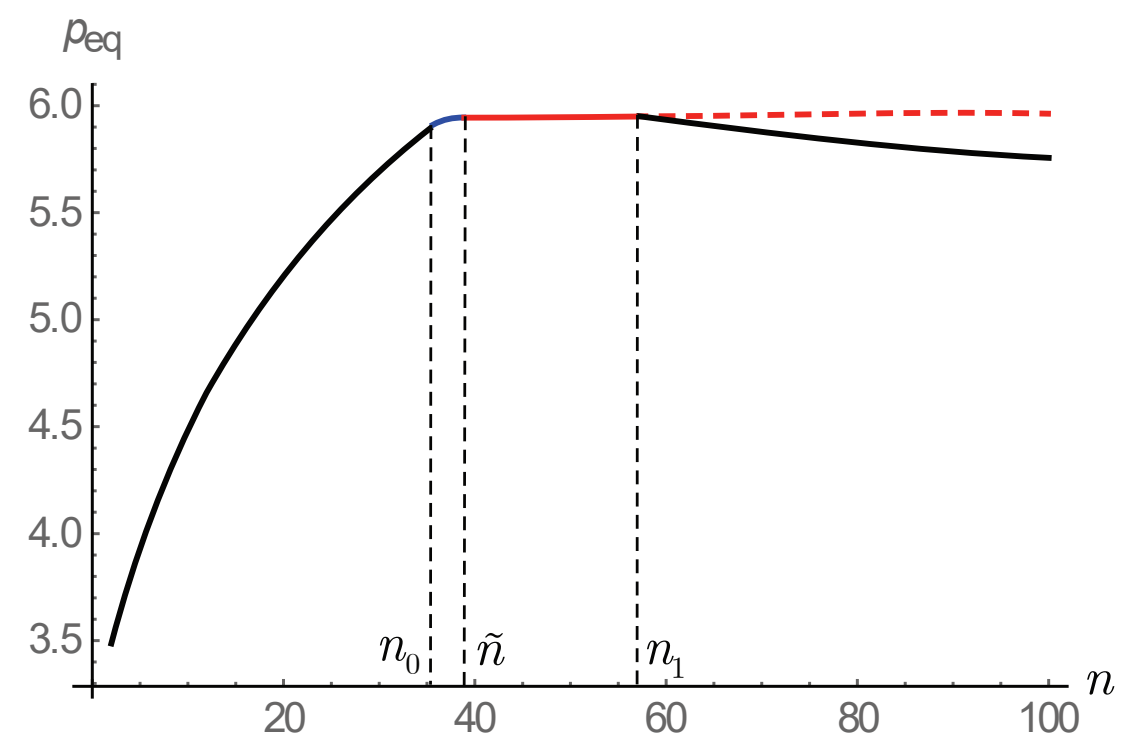

Figure 5: Effect of the number of firms on equilibrium prices $\left(\rho=1.02, \alpha=0.7, w_{1}=1\right.$, $\left.w_{2}=1\right)$.

This last figure illustrates a very original property of our general model of price 
competition: there is an equilibrium even when returns to scale are increasing. As already pointed out in the introduction, this result stems from the sequential choice of production factors which makes the short-run cost convex in the second stage whatever the returns to scale. With a homogeneous single product, the long-run cost is clearly subadditive when returns are increasing. But in our model, in contrast with Dastidar's $2011 b$, this does not entail the existence of an equilibrium because the production factors are chosen sequentially and because the fixed factor $\mathrm{z}$ is set endogenously. It is noteworthy that the case of increasing returns to scale (or more generally, of cost subadditivity) is usually associated with the notion of a natural monopoly. In the contestable market theory (Baumol and Willig, 1981; Baumol et al., 1988), the threat of entry is assumed to be sufficient to drive the price down to the average cost with a single firm operating in the market. As pointed out by Tirole (1988) (p. 310) contestable market theory has been seen as a "generalisation of Bertrand competition to markets with increasing returns to scale". Our general model of price competition with soft capacity constraints clearly refutes this claim. Not only can more than one firm operate in the market when returns are increasing, but they do so with positive profits and potentially high markups. Thus, in presence of increasing returns to scale, a market driven by price competition with soft capacity constraints suffers simultaneously from market inefficiency (existence of a markup) and production inefficiency (the average cost is not minimum).

The same general pattern occurs in all the different cases of returns to scale that has already been analyzed for constant returns to scale. Because of a "capacity effect", before a threshold number of firms, $\tilde{n}$, is reached, the price increases with the number of firms. Beyond this threshold, prices can decrease both when the returns to scale are decreasing (because of the collusive nature of the outcome) and when they are increasing (because of the combined effect of the non-existence of limit pricing strategies and technological inefficiencies). The general model of price competition that we propose accounts for high markups that can increase with the number of firms. Gabaix et al. 
(2016) obtained the same pattern for a homogeneous-good market but based on a very different model with random utility. These authors surveyed the empirical literature on this phenomenon.

\section{Conclusion}

The general model of price competition with soft capacity constraints we propose is a simple and natural extension of existing studies of price competition that bridges three lines of literature: capacity constraints, cost convexity and limit pricing strategies. We show that an equilibrium prediction in pure strategies exists whatever the number of firms and the nature of the returns to scale. This equilibrium prediction is characterized by high markups and prices that can increase with the number of firms in the market. It is the balance between the "natural" feature of this extension and its paradoxical outcome that is the principle contribution of our work.

As economists, we have been taught that price competition is stronger than quantity competition and also that tacit collusion can only result from threats and retaliation in a dynamic setting when firms are not too numerous. Our model clearly undermines these ideas in a very general framework, starting from a production function that is only required to be quasi-concave with decreasing factor marginal productivity.

The core property of the model is summarized in Propositions 1 and 2 in section 3 . The predictable non-cooperative outcome of a non-repeated game of price competition with soft capacity constraints is equivalent to the solution of a joint profit maximization program.

This result is very unusual and it is important to understand the special mechanism that operates behind the scene. In the second stage, for a given soft capacity (i.e. a given level of the fixed factor), because of the Dastidarian property of the model stemming from a convex short-run cost function, there is a continuum of Nash Equilibria in prices. A direct reverse implication is that there is a continuum of levels of the fixed 
factor (chosen in the first stage) that lead to the same price equilibrium in the second. Thus, for a given price in the second stage, it can be profitable for firms to deviate in the first from any fixed factor level that is not the one that maximizes the profit for that price, as long as this price remains sustainable as a Nash equilibrium in the second stage. It is thus the combination of a sequential choice of production factors with the continuum of Nash equilibria in the second stage that is at the origin of the joint profit maximisation property described in Proposition 3.

This has two important consequences. First, from a positive point of view, this property can be used to compute the equilibrium of the game. In general, studies of price competition are cursed by discontinuities, making it impossible to use standard reasoning based on continuous reaction functions to compute the equilibrium. The general methodology provided in section 4 offers a much more tractable method to compute the predictable outcome of the price competition game. Second, from a normative point of view, this outcome, which can be termed "collusive", is obtained in a non-cooperative framework, generalizing the claim of Cabon-Dhersin and Drouhin (2014) that the model offers an alternative mechanism for tacit collusion.

However, as general it is, our model relies on a number of assumptions that are debatable. Of course, as is always the case for Bertrand-Dastidar competition, the assumption that firms are committed to satisfying all incoming demand is a limit that has been commented upon at length. On the one hand, if we consider, as Dixon (1990), that there are hidden costs to turn consumer away, the prediction of a high level of price should be observable in practice. On the other hand, the model allows to discuss, from a more normative point of view, the consequences of including, in a regulated market an obligation for the firms to serve all the incoming demand. In this case, the negative effect of higher prices should be put in balance with potential gains for consumers for not being rationed.

The question of the robustness of our results with respect to some of the simplifying assumptions we made can be considered. What happens when firms use different 
technologies. What happens when a new firm arrives on an existing market? What happens if uncertainty (about demand, costs, etc.) is introduced into the model? What happens if dynamic effects are included? The textbook example that we provide here is sufficiently striking to prove that it is worth pursuing.

\section{Appendix A- Proof of Lemma 1 to 5}

\section{Proof of Lemma 1}

Implicit differentiation of $\hat{v}$ yields Equations (2) to (6).

The quasi-concavity of $f$ means that:

$$
-\hat{v}_{z z} \hat{v}_{y}^{2}+2 \hat{v}_{z y} \hat{v}_{z} \hat{v}_{y}-\hat{v}_{y y} \hat{v}_{z}^{2}=f_{z z} f_{v}<0
$$

This proves that $v$ is quasi-convex.

Moreover, it is easy to check that:

$$
\left|\begin{array}{ll}
\hat{v}_{y y} & \hat{v}_{y z} \\
\hat{v}_{z y} & \hat{v}_{z z}
\end{array}\right|=\frac{1}{f_{v}^{4}}\left|\begin{array}{cc}
f_{z z} & f_{z v} \\
f_{v z} & f_{v v}
\end{array}\right|
$$

If $f$ is concave then this determinant is necessarily positive. The second order pure derivatives of $\hat{v}$ are also positive (cf. (4) and (5)), proving part 2) of the Lemma.

\section{Proof of Lemma 2}

The first step is to expand $\Omega$ and $\Omega_{p}$

$$
\Omega(p, z, m)=\left(\frac{m-1}{m}\right) D(p) p-w_{2}\left(\hat{v}(D(p), z)-\hat{v}\left(\frac{D(p)}{m}, z\right)\right)
$$


Differentiating gives:

$$
\begin{aligned}
& \Omega_{p}(p, z, m) \\
& \quad=\left(\frac{m-1}{m}\right) D(p)+D^{\prime}(p)\left[p-w_{2} \hat{v}_{y}(D(p), z)-\frac{1}{m}\left(p-w_{2} \hat{v}_{y}\left(\frac{D(p)}{m}, z\right)\right)\right]
\end{aligned}
$$

We are now going to prove existence.

For a given $z$ and $m, \Omega(0, z, m)=-w_{2}\left(\hat{v}\left(Q_{\max }, z\right)-\hat{v}\left(\frac{Q_{\max }}{m}, z\right)\right)<0$ (because $\hat{v}_{y}>$ 0 and $\left.Q_{\max }>Q_{\max } / m\right)$. We also have $\Omega\left(p_{\max }, z, m\right)=0$ with $\Omega_{p^{-}}\left(p_{\max }, z, m\right)=$ $D^{\prime-}\left(p_{\max }\right) \frac{m-1}{m} p_{\max }<0$ (with $D^{\prime-}$ being the left derivative of the demand function). $\Omega$ is continuous in $p$ over the interval $\left[0, p_{\max }\right]$, initially negative and finally converging to zero from above. This implies that there is necessarily a $\bar{p}(z, m) \in\left(0, p_{\max }\right)$ that solves $\Omega(p, z, m)=0$

We now prove the uniqueness of $\bar{p}(z, m)$ in $\left(0, p_{\max }\right)$. Over this interval, we have $D(p)>D(p) / m>0$. Moreover, the strict convexity of $\hat{v}$ implies that:

$$
\hat{v}_{y}\left(\frac{D(p)}{m}, z\right)<\frac{\hat{v}(D(p), z)-\hat{v}\left(\frac{D(p)}{m}, z\right)}{D(p)-\frac{D(p)}{m}}<\hat{v}_{y}(D(p), z)
$$

From the definition of $\bar{p}, \frac{m-1}{m} D(\bar{p}) \bar{p}=w_{2}\left(\hat{v}(D(\bar{p}), z)-\hat{v}\left(\frac{D(\bar{p})}{m}, z\right)\right)$ and thus

$$
w_{2} \hat{v}_{y}\left(\frac{D(\bar{p})}{m}, z\right)<\bar{p}<w_{2} \hat{v}_{y}(D(\bar{p}), z)
$$

Finally, considering Equation (15), it is now obvious that $\Omega_{p}(\bar{p}, z, m)>0$. This means that, in the interval $\left(0, p_{\max }\right), \Omega$ can only intercept the $\mathrm{x}$-axis from below. And since $\Omega$ is a continuous functions, this can only happen once.

\section{Proof of Lemma 3}

It is easy to check that $\hat{\pi}(0, z, m)<-w_{1} z, \hat{\pi}\left(p_{\max }, z, m\right)=-w_{1} z$ and $\hat{\pi}_{p^{-}}\left(p_{\max }, z, m\right)<$

0 . Then, the strict concavity of $\hat{\pi}$ in $p$ implies that $\hat{p}$ exists and is unique. Implicit 
differentiation of $\hat{\pi}$ for a given $z$ yields

$$
\left.\frac{\mathrm{d} \hat{p}}{\mathrm{~d} m}\right|_{d z=0}=\frac{1}{m} \frac{D(\hat{p})\left(\hat{p}-w_{2} \hat{v}_{y}\left(\frac{D(\hat{p})}{m}, z\right)\right)}{D(\hat{p})+D^{\prime}(\hat{p})\left(\hat{p}-w_{2} \hat{v}_{y}\left(\frac{D(\hat{p})}{m}, z\right)\right)}<0
$$

For $p<p_{\max }$, we have $\frac{D(p)}{m}>D\left(p_{\max }\right)=0$. The strict convexity of $\hat{v}$ then implies that $\hat{v}\left(\frac{D(p)}{m}, z\right)-0<\left(\frac{D(p)}{m}-0\right) \hat{v}_{y}\left(\frac{D(p)}{m}, z\right)$. By definition, $\hat{p}$ is such that $\hat{p} \frac{D(\hat{p})}{m}=w_{2} \hat{v}\left(\frac{D(\hat{p})}{m}, z\right)$ and then $\hat{p}<w_{2} \hat{v}_{y}\left(\frac{D(\hat{p})}{m}, z\right)$, which gives the sign of the implicit derivative and proves that $\hat{p}$ decreases with $m$. Thus, for $m \geq 2, \hat{p}(z, m)<\hat{p}(z, 1)$ and $\hat{\pi}(\hat{p}(z, m), z, 1)<-w_{1} z$. It follows that for $m \geq 2, \Omega(\hat{p}(z, m), z, m)<0$, implying $\hat{p}(z, m)<\bar{p}(z, m)$.

\section{Proof of Lemma 4}

It is easy to verify that $\hat{\pi}_{p}(0, z, m)>0$ and $\hat{\pi}_{p^{-}}\left(p_{\max }, z, m\right)<0 . \hat{\pi}_{p}$ is continuous, ensuring that the program has an interior maximum. The strict concavity of $\hat{\pi}$ with $p$ ensures that the maximum is unique. Because $\hat{\pi}_{p}(\hat{p}, z, m)>0, p^{*}(z, m)>\hat{p}(z, m)$

\section{Proof of Lemma 5}

At $\hat{p}, \frac{D(\hat{p})}{m} \hat{p}-w_{2} \hat{v}\left(\frac{D(\hat{p})}{m}, z\right)=0$.

The derivative of the above expression with respect to $z$ is:

$$
\left.\frac{\mathrm{d} \hat{p}}{\mathrm{~d} z}\right|_{d m=0}=\frac{w_{2} \hat{v}_{z}\left(\frac{D(\hat{p})}{m}, z\right)}{\frac{D^{\prime}(\hat{p})}{m} \hat{p}+\frac{D(\hat{p})}{m}-w_{2} \frac{D^{\prime}(\hat{p})}{m} \hat{v}_{y}\left(\frac{D(\hat{p})}{m}, z\right)}<0
$$

From Equation (17), we have

$$
\left.\frac{\mathrm{d} \hat{p}}{\mathrm{~d} m}\right|_{d z=0}<0
$$

At $\bar{p}$, we have $\Omega(\bar{p}, z, m)=0$. The derivatives of the above equality with respect to $z$ and $m$ are: 


$$
\left.\frac{\mathrm{d} \bar{p}}{\mathrm{~d} z}\right|_{d m=0}=-\frac{\Omega_{z}(\bar{p}, z, m)}{\Omega_{p}(\bar{p}, z, m)}=\frac{w_{2}\left(\hat{v}_{z}(D(\bar{p}), z)-\hat{v}_{z}\left(\frac{D(\bar{p})}{m}, z\right)\right)}{\Omega_{p}(\bar{p}, z, m)}
$$

which is $<0$ since $\Omega_{p}(\bar{p}, z, m)>0$ and $\hat{v}_{z}<0, \hat{v}_{y z}<0$.

$$
\left.\frac{\mathrm{d} \bar{p}}{\mathrm{~d} m}\right|_{d z=0}=-\frac{\Omega_{m}(\bar{p}, z, m)}{\Omega_{p}(\bar{p}, z, m)}=-\frac{\frac{D(\bar{p})}{m^{2}}\left(\bar{p}-w_{2} \hat{v}_{y}\left(\frac{D(\bar{p})}{m}, z\right)\right)}{\Omega_{p}(\bar{p}, z, m)}
$$

which is $<0$ since $\Omega_{p}(\bar{p}, z, m)>0$ and from Equation $(16), \bar{p}>w_{2} \hat{v}_{y}\left(\frac{D(\bar{p})}{m}, z\right)$.

Finally, we obtain,

$$
\left.\frac{\mathrm{d} p^{*}}{\mathrm{~d} z}\right|_{d m=0}=-\frac{\hat{\pi}_{p z}\left(p^{*}, z, m\right)}{\hat{\pi}_{p p}\left(p^{*}, z, m\right)}=w_{2} \frac{D^{\prime}\left(p^{*}\right)}{m} \frac{\hat{v}_{y z}\left(\frac{D\left(p^{*}\right)}{m}, z\right)}{\hat{\pi}_{p p}\left(p^{*}, z, m\right)}<0
$$

and

$$
\left.\frac{\mathrm{d} p^{*}}{\mathrm{~d} m}\right|_{d z=0}=-\frac{\hat{\pi}_{p m}\left(p^{*}, z, m\right)}{\hat{\pi}_{p p}\left(p^{*}, z, m\right)}=-w_{2} \frac{D^{\prime}\left(p^{*}\right)}{m^{3}} \frac{D\left(p^{*}\right) \hat{v}_{y y}\left(\frac{D\left(p^{*}\right)}{m}, z\right)}{\hat{\pi}_{p p}\left(p^{*}, z, m\right)}<0
$$

\section{Appendix B- Proof of Proposition 1}

\section{Methodological statement}

1. Price competition is cursed by discontinuity of the payoff function in the vicinity of the equilibrium. In the literature, the use of mixed strategies solve partially the problem. Dasgupta and Maskin (1986) provide some results that, relying on weaker forms of continuity, prove the existence and uniqueness of mixed strategies equilibria for certain class of discontinuous game that can be applied to price competition with capacity constraint. In this paper, we address the problem of the existence of pure strategies equilibria in line with Dastidar (1995). We want to point out the methodological switch he proposes, restarting the reasoning from scratch, relying only on the definition of Nash Equilibrium, a definition that 
requires no assumption of any kind for continuity of the payoff function. ${ }^{8}$ Within this approach, the only requirement is to build a clever partition of the outcome space, that allows to test for a finite number of class of unilateral profitable deviations from any possible outcome. To solve the equilibrium in the second stage, we adopt exactly the same line of reasoning, and the purpose of Lemma 2 to 5 is to provide this "clever" partition of the outcomes space.

2. However, conversely to Dastidar (1995), as most models of capacity constraint, our model is a sequential two stage game, with firms playing simultaneously at each stage, i.e. a game of imperfect information. Moreover, conversely to the approach in mixed strategies, we have a multiplicity of the equilibria in the second stage instead of uniqueness, a property that makes impossible to reduce the whole game to a simple strategic one. Our innovation to resolve this problem is thus to extend the Dastidarian methodological move to the case of Subgame Perfect Nash Equilibrium.

3. We know that, in general, in a sequential game of complete but imperfect information, when we have multiplicity of equilibria in some subgame, subgame perfection may failed to achieve sensible equilibrium prediction. It is usual in this case to introduce more sophisticated equilibrium concept and refinement relying on some kink of belief or expectation rules, etc.. However in our special setting, despite the multiplicity of equilibria, some subgame perfect equilibria exists and we will base our equilibrium prediction on this underlying concept.

\section{Core of the proof of Proposition 1}

An outcome $\left(\vec{z}^{e}, \vec{p}^{e}\right)$ with $\vec{z}^{e}=\left(z_{1}^{e}, \cdots, z_{n}^{e}\right)$ and $\vec{p}^{e}=\left(p_{1}^{e}, \cdots, p_{n}^{e}\right)$ is a Subgame Perfect Nash Equilibrium if and only if:

\footnotetext{
${ }^{8}$ It is also important to emphasise that many considerations on the existence on the equilibrium in price competition with pure strategies take their roots in Edgeworth (1925), in a time in which the notion of Nash Equilibrium does not even exist.
} 
1. $\left(\overrightarrow{\mathbf{z}}^{e}, \vec{p}^{e}\right)$ is an Nash equilibrium in price in the second stage (the bold notation indicates that the value is given at the stage of reasoning):

$$
\forall i \in\{1, \cdots, n\}, \forall p_{i} \in\left[0, p_{\max }\right], \pi_{i}\left(p_{i}^{e}, \overrightarrow{\mathbf{p}}_{-i}^{e}, \overrightarrow{\mathbf{z}}^{e}\right) \geq \pi_{i}\left(p_{i}, \overrightarrow{\mathbf{p}}_{-i}^{e}, \overrightarrow{\mathbf{z}}^{e}\right)
$$

and 2. $\left(\vec{z}^{e}, \vec{p}^{e}\right)$ is an Nash equilibrium of the whole game:

$$
\forall i \in\{1, \cdots, n\}, \forall p_{i} \in\left[0, p_{\max }\right], \forall z_{i} \in \mathbb{R}^{+}, \pi_{i}\left(\left(z_{i}^{e}, p_{i}^{e}\right), \overrightarrow{\mathbf{z}}_{-i}^{e}, \overrightarrow{\mathbf{p}}_{-i}^{e}\right) \geq \pi_{i}\left(\left(z_{i}, p_{i}\right), \overrightarrow{\mathbf{z}}_{-i}^{e}, \overrightarrow{\mathbf{p}}_{-i}^{e}\right)
$$

We are interested in outcomes in which all $n$ firms operate in the market, meaning that we consider the possibility for an outcome in which all firms are setting the same price $p^{N}$ to be $S P N E$.

Step 1: $p^{N}$ has to be a Nash equilibrium in the second stage. The firms have no incentive to unilaterally deviate in the second stage as long as $p^{N} \in \bigcap_{i}\left[\hat{p}\left(z_{i}, n\right), \bar{p}\left(z_{i}, n\right)\right] \neq$ $\emptyset$. In other words the necessity for the outcome to be a Nash equilibrium in price in the second stage prevent the possibility for the firms to play too different levels of fixed factor in the first stage. More precisely, for all $i, z_{i}$ has to belong in the interval $\left[\bar{z}\left(p^{N}, n\right), \hat{z}\left(p^{N}, n\right)\right]$ with $\bar{z}\left(p^{N}, n\right)$ such that $\bar{p}(z, n)=p^{N}$ and $\hat{z}\left(p^{N}, n\right)$ such that $\hat{p}(z, n)=p^{N}$ (that are both unique, thanks to Lemma 5$)$.

Among all the outcomes that are Nash equilibria in the second stage (Step 1), with all firms operating the market, we are now going to eliminate those who are subject to profitable unilateral deviation in stage 1. Because the strategy of player $i$ in stage 1 is of dimension 2, firms can deviate in either dimension or both and we will have to consider all the possible deviations.

Step 2: By definition of program $\mathcal{P}_{1}(p, n)$ :

$$
\begin{aligned}
& \forall i \in\{1, \cdots, n\}, \forall z_{i}, z_{-i} \in\left[\bar{z}\left(p^{N}, n\right), \hat{z}\left(p^{N}, n\right)\right], \\
& \quad \pi_{i}\left(\left(z^{*}\left(p^{N}, n\right), p^{N}\right), \overrightarrow{\mathbf{z}}_{-i}, \overrightarrow{\mathbf{p}}_{-i}^{N}\right) \geq \pi_{i}\left(\left(z_{i}, p^{N}\right), \overrightarrow{\mathbf{z}}_{-i}, \overrightarrow{\mathbf{p}}_{-i}^{N}\right)
\end{aligned}
$$


It means that for firm $i$, the strategy $\left(z^{N}, p^{N}\right)$ (with $z^{N}=z^{*}\left(p^{N}, n\right)$ ) played in the first stage dominates all other strategy that lead to $p^{N}$ being a Nash Equilibria in the second stage with all firm operating the market. All the firms having the same technology, we conclude that a necessary condition for an outcome of the game with all $n$ firms quoting $p^{N}$ to be a $S P N E$ is that all firms choose the same level of fixed factor $\left(z^{*}\left(p^{N}, n\right)\right.$ solution of $\mathcal{P}_{1}(p, n)$. That correspond to the first condition (i.e. optimality condition) of Proposition 1.

Step 3: Now we are going to test if an outcome in which all firms play $\left(z^{*}\left(p^{N}, n\right), p^{N}\right)$ is subject to profitable unilateral deviation $\left(z^{d}, p^{d}\right)$ by firm $i$, with $p^{d} \neq p^{N}$, under condition that $p^{d}$ can be sustained as a Nash equilibrium in the second stage (not necessarily with all firms operating the market).

Step 3.1 Let us start by all the unilateral deviation such that: $z^{d} \in \mathbb{R}^{+}$and $p^{d} \in\left(p^{N}, p_{\max }\right]$. In this case the firm sells nothing and the profit is $-w_{1} z^{d}$. The "best" of these unilateral deviation is $z^{d}=0$ that earns zero profit. This deviation is not profitable as long as $\hat{\pi}\left(p^{N}, z^{N}, n\right) \geq 0$. That impose the second condition (i.e. profitability) of Proposition 1

Step 3.2 Let us start by all the unilateral deviation such that: $z^{d} \in \mathbb{R}^{+}$and $p^{d} \in$ $\left[0, \hat{p}\left(z^{N}, n\right)\right)$. In this case the firm will operate the market alone in the second stage. The profit corresponding to these unilaterally deviating strategies are upper bounded by profit corresponding to $\left.\left(\underset{z}{\operatorname{argmax}}, \hat{\pi}\left(\hat{p}\left(z^{N}, n\right), z, 1\right), 1\right), \hat{p}\left(z^{N}, n\right)\right)$. That correspond to the third condition (i.e. non-existence of limit pricing strategy) of Proposition 1

All those conditions are necessary for having a SNPE and must be satisfied simultaneously.

\section{Core of the proof of Proposition 2}

We start with an outcome that verifies all the three conditions of Proposition 1 and we consider remaining possible deviations. 
Step 3.3 Finally, let us consider the case with $p^{d} \in\left[\hat{p}\left(z^{N}, n\right), p^{N}\right)$ and $z^{d} \in \mathbb{R}+$. The case $z^{d}<\bar{z}\left(p^{d}, n\right)$ or $z^{d}>\hat{z}\left(p^{N}, n\right)$ can be excluded because they are incompatible with the possibility for $p^{d}$ to be sustain as a Nash equilibrium in the second stage. For a given $p^{d}$, all the remaining unilateral deviations of that kind are dominated by $\left(z^{*}\left(p^{d}, n\right), p^{d}\right)$. Thus as long as: $\forall p \in\left[\hat{p}\left(z^{N}, n\right), p^{N}\right), \quad \pi\left(p^{N}, z^{*}\left(p^{N}, n\right), n\right) \stackrel{\text { def }}{=} \Pi\left(p^{N}, n\right) \geq$ $\pi\left(p, z^{*}(p, n), n\right) \stackrel{\text { def }}{=} \Pi(p, n)$ these unilateral deviations are unprofitable.

Step 4: When the supplementary condition of Proposition 2 is satisfied, Step 2, 3.1, 3.2, 3.3 define necessary conditions for $\left(z^{N}, p^{N}\right)$ to be a $S P N E$ with all firms operating the market. But taken together they exhausts all the possible case implying that they are also sufficient.

Remark: The condition described in case 3.3 is operative only for outcomes associated with the non-decreasing part (in $p$ ) of the function П. For outcomes associated with the decreasing part (in $p$ ) of $\Pi$ subgame perfection is not powerful enough to obtain an equilibrium, even if those outcomes can be appropriately ranked relying on function $\Pi$. And of course they will be dominated by the outcome that is associated with the price $p$ that maximizes $\Pi$ for a given $n$, which is a SPNE. That will be the core argument of proposition 3.

\section{References}

Bagh, A. (2010). Pure strategy equilibria in bertrand games with discontinuous demand and asymmetric tie-breaking rules. Economics letters 108, 277-279.

Baumol, W. J., J. C. Panzar, R. D. Willig, E. E. Bailey, D. Fischer, and D. Fischer (1988). Contestable markets and the theory of industry structure, Volume 169. Harcourt Brace Jovanovich New York.

Baumol, W. J. and R. D. Willig (1981). Fixed costs, sunk costs, entry barriers, and sustainability of monopoly. The Quarterly Journal of Economics 96(3), 405-431. 
Baye, M. and D. Kovenock (2008). Bertrand competition. The New Palgrave Dictionary of Economics 1, 476-480.

Baye, M. R. and J. Morgan (1999). A folk theorem for one-shot bertrand games. Economics Letters 65(1), 59 - 65.

Boccard, N. and X. Wauthy (2000). Bertrand competition and Cournot outcomes: further results. Economics Letters 68(3), 279-285.

Boccard, N. and X. Wauthy (2004). Bertrand competition and Cournot outcomes: a correction. Economics Letters 84(2), 163-166.

Burguet, R. and J. Sákovics (2017). Bertrand and the long run. International Journal of Industrial Organization 51, 39-55.

Cabon-Dhersin, M.-L. and N. Drouhin (2014). Tacit collusion in a one-shot game of price competition with soft capacity constraints. Journal of Economics $\& 5$ Management Strategy 23(2), 427-442.

Chamberlin, E. (1933). The theory of monopolistic competition. Cambridge: Harverd University Press.

Chaudhuri, P. R. (1996). The contestable outcome as a bertrand equilibrium. Economics Letters 50(2), 237-242.

Chen, Y. and M. H. Riordan (2008). Price-increasing competition. The RAND Journal of Economics 39(4), 1042-1058.

Chowdhury, P. R. (2002). Limit-pricing as bertrand equilibrium. Economic Theory $19(4), 811-822$.

Chowdhury, P. R. (2009). Bertrand competition with non-rigid capacity constraints. Economics Letters 103(1), 55 - 58. 
Chowdhury, P. R. and K. Sengupta (2004). Coalition-proof Bertrand equilibria. Economic Theory $24(2), 307-324$.

Dasgupta, P. and E. Maskin (1986). The existence of equilibrium in discontinuous economic games, i: Theory. The Review of economic studies 53(1), 1-26.

Dastidar, K. G. (1995). On the existence of pure strategy Bertrand equilibrium. Economic Theory 5, 19-32.

Dastidar, K. G. (2001). Collusive outcomes in price competition. Journal of Economics 73(1), 81-93.

Dastidar, K. G. (2011a). Bertrand equilibrium with subadditive costs. Economics Letters 112, 202-204.

Dastidar, K. G. (2011b). Existence of Bertrand equilibrium revisited. International Journal of Economic Theory 7, 331-350.

Davidson, C. and R. Deneckere (1986). Long-term competition in capacity, shortrun competition in price, and the cournot model. Rand Journal of Economics 17, $404-415$.

Dixit, A. (1980). The role of investment in entry-deterrence. The economic journal $90(357), 95-106$.

Dixon, H. (1987). Approximate bertrand equilibria in a replicated industry. The review of economic studies 54(1), 47-62.

Dixon, H. (1990). Bertrand-Edgeworth equilibria when firms avoid turning customers away. The Journal of Industrial Economics 39(2), 131-146.

Edgeworth, F. (1925). The pure theory of monopoly. Papers Relating to Political Economy 1, 111-42. 
Gabaix, X., D. Laibson, D. Li, H. Li, S. Resnick, and C. G. de Vries (2016). The impact of competition on prices with numerous firms. Journal of Economic Theory 165, 124 .

Harrington, J. E. (1989). A re-evaluation of perfect competition as the solution to the bertrand price game. Mathematical Social Sciences 17(3), 315-328.

Hoernig, S. H. (2002). Mixed Bertrand equilibria under decreasing returns to scale: an embarrassement of riches. Economic Letters 74, 359 - 362.

Hoernig, S. H. (2007). Bertrand games and sharing rules. Economic Theory 31(3), $573-585$.

Kreps, D. and J. Scheinkman (1983). Quantity precommitment and Bertrand competition yield Cournot outcomes. Bell Journal of Economics 13, 111-122.

Maggi, G. (1996). Strategic trade policies with endogenous mode of competition. The American Economic Review 86(1), pp. 237-258.

Novshek, W. and P. R. Chowdhury (2003). Bertrand equilibria with entry: limit results. International Journal of Industrial Organization 21(6), 795 - 808.

Rosenthal, R. W. (1980). A model in which an increase in the number of sellers leads to a higher price. Econometrica, 1575-1579.

Routledge, R. (2010). Bertrand competition with cost uncertainty. Economics Letters 107, 356-359.

Saporiti, A. and G. Coloma (2010). Bertrand competition in markets with fixed costs. The BE Journal of Theoretical Economics 10(1), 27.

Spence, A. M. (1977). Entry, capacity, investment and oligopolistic pricing. The Bell Journal of Economics, 534-544.

Spulber, D. F. (1989). Regulation and markets. MIT press. 
Tirole, J. (1988). The Theory of Industrial Organization. Cambridge MA: The MIT Press.

Vives, X. (1999). Oligopoly Pricing, old ideas and new tools. Cambridge MA: The MIT Press.

Yano, M. and T. Komatsubara (2017). Price competition or price leadership. Economic Theory, 1-35. 